\title{
Re-Demokratisierung der Postdemokratie durch alternative Beteiligungsverfahren?'
}

\author{
Dirk Jörke
}

\section{Re-democratization of Post-democracy by Alternative Procedures of Participation?}

Abstract: Since some years political scientists observe a growing political dissatisfaction. On closer inspection one sees that mainly socially disadvantaged citizens have retreated from the democratic process. This applies not only to the participation in elections and membership in political parties, but to a greater degree also to alternative forms of political participation such as town meetings and referenda. Against this background, the article is looking for possibilities of a reintegration of socially disadvantaged citizens into the democratic process. Following considerations made by Young and McCormick, who highlight an "aristocratic effect" of formal democratic procedures, a suspensive veto-right for socially disadvantaged citizens is proposed at the end.

Keywords: Post-democracy, social disadvantage, procedures of participation, theory of democracy

Schlagwörter: Postdemokratie, soziale Benachteiligung, Beteiligungsverfahren, Demokratietheorie

\section{Einleitung}

Moderne westliche Demokratien können auf eine beachtliche Erfolgsgeschichte zurückblicken. Ihnen ist es nicht nur gelungen, ihren Bürgern grundlegende liberale Grundrechte zu gewähren, sondern sie erzielten darüber hinaus auch eine erhebliche Integration der Bevölkerung. Insbesondere konnten diese politischen Regime den Gegensatz zwischen Arbeit und Kapital befrieden. Wesentlich hierfür war die politische Integration der Arbeiterklasse durch das allgemeine Wahlrecht. Zwar ist das Ideal der gleichen Partizipationsmöglichkeiten immer nur ein Versprechen gewesen, das nie vollständig eingelöst werden konnte (Bobbio 1988). Das allgemeine Wahlrecht hat aber in westlichen Gesellschaften zumindest dazu beigetragen, dass die politischen Eliten sich an dem Willen der Mehrheit der Wähler orientiert haben. Die Folgen hiervon waren der Ausbau der sozialen Sicherungssysteme und damit die Annäherung an das Ideal einer sozialen Demokratie sowie eine hohe Zufriedenheit mit der Demokratie und ihren Repräsentanten (Crouch 2004; Meyer 2009).

1 Für sehr hilfreiche Kommentare und Hinweise möchte ich mich bei Michael Hein, Thomas Lenz und Tobias Müller, den TeilnehmerInnen des Autorenworkshops und des Seminars zur „Postdemokratie“ an der Technischen Universität Darmstadt sowie ganz besonders bei den beiden anonymen GutachterInnen bedanken. 
Allerdings mehren sich die Indizien, dass sich diese Erfolgsgeschichte erschöpft haben könnte. In westlichen Demokratien lässt sich seit etwa 20 Jahren ein erheblicher Rückgang der Unterstützung der grundlegenden politischen Institutionen und ihrer Repräsentanten seitens der Bürgerinnen und Bürger beobachten, der sich am deutlichsten in Phänomenen wie Politikverdrossenheit, der mangelnden Bereitschaft zur Mitarbeit in Parteien und nicht zuletzt der abnehmenden Wahlbeteiligung niederschlägt (Putnam u. Pharr 2000; Dalton 2004; Nève 2009). Zudem zeigt sich, dass es insbesondere sozial schwache Bürger sind, die den Gang zur Urne verweigern und sich aus den Parteien zurückgezogen haben (Schäfer 2010; Merkel u. Petring 2011). ${ }^{2}$ Dieser Rückzug, der sich auch als Reaktion auf die nachlassende Integrationskraft westlicher Gesellschaften deuten lässt, ist - so die Ausgangsthese - als eine ernstzunehmende Gefährdung der Demokratie und als ein Merkmal einer postdemokratischen Konstellation anzusehen. Postdemokratisch sind die gegenwärtigen Verhältnisse also nicht allein aufgrund der Verlagerung von demokratischen Entscheidungsprozessen auf die supranationale Ebene und die wachsende Macht nicht-demokratisch legitimierter Akteure, sondern auch deshalb, weil die Beteiligung an demokratischen Verfahren einen deutlichen Bias aufweist. ${ }^{3}$

Vor diesem Hintergrund möchte ich in diesem Artikel Wege diskutieren, wie sozial schwache Milieus wieder stärker in den demokratischen Prozess inkludiert werden können. Im Zentrum steht die Frage der Erneuerung und Ergänzung der repräsentativen Demokratie. Dabei beschränke ich mich auf institutionelle Aspekte, also auf Rechte und Verfahren politischer Beteiligung. Nicht explizit thematisiert werden hingegen gesellschaftstheoretische Aspekte, auch wenn diese selbstverständlich den Hintergrund der nachfolgenden Ausführungen abgeben. ${ }^{4}$ Es geht an dieser Stelle also nicht um die Verortung der Exklusionsprozesse im Rahmen einer soziologischen Theorie der Postdemokratisierung, wie sie etwa Ingolfur Blühdorn (2013, S. 126) mit seinem „starken Begriff der Postdemokratie“ entwickelt hat. Dahinter steht auch die Überzeugung, dass Institutionen im Allgemeinen und Verfahren der politischen Beteiligung im Besonderen durchaus einen Unterschied bewirken können, sie also nicht bloße ,Überbauphänomene‘ darstellen.

Im folgenden zweiten Abschnitt werde ich zunächst drei Gründe anführen, weshalb der Rückzug sozial schwacher Milieus aus den demokratischen Verfah-

2 Ich werde in diesem Artikel die Ausdrücke, sozial Schwache‘, ,sozial Benachteiligte‘ sowie ,sozial schwache' bzw. ,unterprivilegierte Milieus' synonym verwenden und beziehe mich dabei auf Michael Vester, der zu diesem gesellschaftlichen Milieu 27\% der deutschsprachigen Wohnbevölkerung zählt und es wie folgt definiert: „Es vereint Verlierer der ökonomischen Modernisierung, die vor allem aus den kleinbürgerlichen und unterprivilegierten Milieus stammen, insbesondere ältere und teilweise auch jüngere Menschen mit wenig Bildungskapital und unsicheren Zukunftsperspektiven" (2009, S. 53).

3 Crouch fasst dies als „a weakening of the political importance of ordinary working people“ (2004, S. 29) zusammen. Zur Postdemokratiedebatte vgl. die Beiträge in Buchstein u. Nullmeier (2006) sowie in Nordmann et al. (2012).

4 Darauf, dass es „schlechthin neben der Sache liegend“ ist, eine „politikwissenschaftliche Demokratietheorie ohne Bezug auf Wirtschaft als politisch-gesellschaftliche Tätigkeit zu betreiben “, hat jüngst zu Recht Wolfgang Streeck (2013, S. 17) hingewiesen. Dem wird hier insofern entsprochen, als es gerade die gesellschaftlichen Effekte ökonomischer Dynamiken sind, denen demokratietheoretisch begegnet werden soll. 
ren als problematisch anzusehen ist (2). Anschließend erfolgt eine Auseinandersetzung mit Deutungen von Pierre Rosanvallon, John Keane und Paul Nolte, die weniger einen Bedeutungsverlust als einen Wandel der Demokratie behaupten und eine neue Form einer bürgerschaftlichen „Counter Democracy“ (Rosanvallon 2008) oder „monitory democracy“ (Keane 2009) im Entstehen sehen. Es wird sich jedoch zeigen, dass die praktische Umsetzung dieser Demokratiekonzeptionen den Trend zur Exklusion sozial Benachteiligter mehr verstärkt als dass sie ihm entgegenwirkt (3). Damit stellt sich die Frage nach Verfahren, die einen derartigen Bias vermeiden. Ich werde die Vorschläge einer Wahlpflicht (4.1), von gelosten Versammlungen (4.2) und von Mechanismen der Gruppenrepräsentation (4.3) diskutieren. Alle drei zielen auf die Realisierung einer deskriptiven Repräsentation. Allerdings stoßen auch diese Vorschläge an Grenzen, die sich aus der unterschiedlichen Verteilung politischer Ressourcen und Kompetenzen in modernen Gesellschaften ergeben. Daher möchte ich im fünften Abschnitt einen eigenen Vorschlag unterbreiten. Zunächst werde ich im Anschluss an Überlegungen von Iris Marion Young, Bernard Manin und John McCormick auf die ,aristokratischen Effekte' formaldemokratischer Verfahren verweisen (5.1). Um dem zu begegnen, schlage ich die Einführung von Vetorechten für sozial Benachteiligte vor (5.2). Am Ende steht ein kurzes Plädoyer für eine experimentelle Demokratie (6).

\section{Warum gleiche Beteiligung in einer Demokratie unhintergehbar ist}

Warum ist der Rückgang der Wahlbeteiligung aus demokratietheoretischer Perspektive problematisch? Kann man nicht das Recht, sich dem Gang zur Wahlurne zu verweigern oder sich nicht für Politik zu interessieren, sich also nicht wie in Autokratien politisch mobilisieren zu lassen, als eine Errungenschaft liberaler Demokratien ansehen? Und lässt sich eine niedrige Wahlbeteiligung nicht auch als Ausdruck einer grundlegenden Zufriedenheit mit dem politischen System deuten? Solche Beschwichtigungen unterschlagen jedoch, dass es, wie einleitend erwähnt, vor allem bildungsferne und einkommensschwache Bürger sind, die sich aus den Verfahren der parlamentarischen Demokratie zurückziehen. Sie engagieren sich kaum in politischen Parteien, gehen seltener zur Wahl und interessieren sich nur wenig für Politik. Verbreitet ist vielmehr die Überzeugung, dass ,die Politiker' sich nicht hinreichend um ihre Interessen und Bedürfnisse kümmern, diese ,zu abgehoben', zu weit entfernt von der Lebenswelt der ,normalen' Bürgerinnen und Bürger sind. In diesen Milieus ist die Politikverdrossenheit entsprechend stark ausgeprägt; diese reicht bisweilen auch bis zur Ablehnung der Demokratie als normative Leitvorstellung (Hay 2007; Embacher 2009). Es spricht einiges dafür, dass diese Einstellungen und der Rückzug aus den demokratischen Verfahren strukturelle Ursachen besitzen: „Soziale und ökonomische sowie bildungsbezogene Ungleichheiten hindern von einer bestimmten Größenordnung an die von ihnen Betroffenen daran, ihre politischen Staatsbürgerrechte aktiv wahrnehmen zu können“ (Meyer 2009, S. 25). Mit Crouch (2004, S. 23-24) ist dabei von einer Passivierung politischer Beteiligung in der Postdemokratie auszugehen, die insbesondere sozial Schwache betrifft. 
Der „Demokratieverdruss“ (Embacher 2009) sozial benachteiligter Bürger ist aus mindestens drei Gründen kritisch zu sehen. Zum Ersten ist aus einer normativen Perspektive für die Demokratie das Prinzip der politischen Gleichheit grundlegend (Dahl 2006; Christiano 1996). Es ist die Vorstellung, dass politische Entscheidungen nur dann legitim sind, wenn alle von ihr Betroffenen die effektive Möglichkeit der Beteiligung haben, wobei es auf dieser grundlegenden Ebene gleichgültig ist, ob diese Beteiligung direkt oder über repräsentative Mechanismen erfolgt. Wenn sich aber spezifische Bevölkerungsgruppen aus strukturellen Gründen nicht mehr in einem hinreichenden Maße an den Prozessen der Entscheidungsfindung beteiligen, dann wird in der Folge auch das Prinzip der politischen Gleichheit unterwandert. Aus diesem Partizipationsdefizit wird in parlamentarischen Demokratien schließlich ein Repräsentationsdefizit, als Parteien nicht im gleichen Maße um die Stimmen und Interessen der sozial Schwachen werben müssen, wie um diejenigen der Mittelschichten (vgl. Merkel u. Petring 2011, S. 12-13; Schäfer 2011, S. 9). ${ }^{5}$ Das führt zum zweiten Grund.

Der Rückzug der sozial Benachteiligten unterminiert das Versprechen der Demokratie, über demokratische Prozeduren zu einer Angleichung der Lebensverhältnisse und der Chancen zwischen den Gesellschaftsmitgliedern, also zu mehr sozialer Gleichheit beizutragen. So haben insbesondere sozialdemokratische Parteien bis in die 1970er-Jahre demokratische Institutionen als Vehikel der Angleichung der sozialen Lebensverhältnisse zu nutzen gewusst (Schmidt 2008, S. 362369; Nolte 2012, S. 437). Demokratische Beteiligung besitzt unter diesem Gesichtspunkt keinen intrinsischen, sondern einen instrumentellen Charakter. Sie dient einem Ziel, welches zwar unabhängig von demokratischen Prinzipien - hier in einem engen Sinne der gleichen Beteiligung verstanden - gerechtfertigt werden kann, dessen Realisierung jedoch, wie die vergleichende Performanzforschung zeigt, auf eine möglichst egalitäre Beteiligung angewiesen ist: Politische Systeme, die eine hohe empirische Inklusivität der politischen Verfahren aufweisen, besit-

5 Es bietet sich an, analytisch zwischen drei Dimensionen der politischen Gleichheit zu unterscheiden: 1. Die gleiche effektive Möglichkeit der Teilnahme an den demokratischen Verfahren, d.h. niemand ist strukturell ausgeschlossen (wie das bei sozial Benachteiligten jedoch der Fall ist). Das möchte ich als den gleichen Zugang bezeichnen. 2. Die gleiche Möglichkeit, die Interessen zu Gehör zu bringen (setzt neben der bloßen Teilnahme auch Fähigkeiten/Ressourcen voraus, um z.B. das Wort zu ergreifen oder einen Zugang zu Massenmedien zu besitzen). 3. Die gleiche Chance, die Interessen auch durchzusetzen, also effektiv Einfluss auszuüben. Ich verdanke diese Differenzierung einem Kommentar von Thomas Lenz. In der politischen Praxis lässt sich freilich nur schwer eindeutig zwischen diesen drei Dimensionen politischer Gleichheit unterscheiden, allerdings setzt Gehör Zugang und Einfluss Gehör voraus. Im vierten und fünften Abschnitt werde ich auf diese Differenzierung zurückkommen. 
zen auch einen höheren Grad an sozialer Gleichheit, die Vermögensunterschiede etwa sind weniger stark ausgeprägt. ${ }^{6}$

Ein dritter Grund besteht darin, dass die mangelnde Integration sozial Schwacher in die demokratischen Verfahren zu Legitimationskrisen bis hin zu einer grundsätzlichen Ablehnung der Demokratie, also nicht nur ihres gegenwärtigen Zustandes, führen kann. Daher sind auch empirische Befunde, dass es vor allem, wenn auch nicht ausschließlich, minderprivilegierte Milieus sind, die rechtspopulistische und rechtsextreme Parteien wählen (Oesch 2008; Vester 2009, S. 54), aus einer demokratietheoretischen Perspektive besonders besorgniserregend. Verfolgen diese Parteien doch zumeist eine Agenda, die sich gegen die Prinzipien der liberalen Demokratie richtet, bis hin zu einer Infragestellung des Minderheitenschutzes und der Rechtsgleichheit (Mudde 2007).

\section{Neue Formen demokratischer Beteiligung?}

Rosanvallon (2008, 2010), Keane (2009) und Nolte (2012) grenzen sich von der Postdemokratiediagnose $a b$ und argumentieren, dass wir in den westlichen Gesellschaften nicht das Ende, sondern vielmehr einen Wandel der Demokratie erleben. Demokratische Beteiligung erfolge heutzutage in unterschiedlichen Kanälen und beschränke sich nicht länger auf traditionelle Formen des Wählens und des Engagements in Parteien. Zu beobachten sei vielmehr eine Ausdifferenzierung, die von Referenden über Mediationsverfahren, dem Engagement in lokalen Bürgerinitiativen und transnationalen Nichtregierungsorganisationen, über die Beteiligung an Demonstrationen bis hin zu einem politisch bewussten Konsum reiche. Ein besonderes Gewicht komme bei dieser „neuen bürgerlichen Interessenwahrung gegenüber dem demokratischen Staat" (Nolte 2012, S. 419) den Gerichten zu, also der Möglichkeit, gegen staatliche Entscheidungen sowie gegen Diskriminierungen zu klagen. Aus einer demokratietheoretischen Perspektive, die sowohl dem Ideal der politischen Gleichheit als auch dem der sozialen Demokratie verpflichtet ist, ist diese Aufwertung alternativer Beteiligungsverfahren jedoch aus zwei Gründen problematisch.

Zum einen zeigen empirische Studien zu den unkonventionellen Formen der Bürgerbeteiligung einen deutlichen sozialen Bias. Dass ich mich an Demonstratio-

6 An anderer Stelle (Jörke 2010) habe ich zwischen zwei Versprechen der Demokratie unterschieden: das intrinsische Versprechen der gleichen Teilhabe am politischen Prozess und das instrumentelle Versprechen, durch demokratische Verfahren die Geschicke der Gesellschaft in Richtung auf mehr soziale Egalität bestimmen zu können. Diese beiden Versprechen lassen sich zwar unabhängig voneinander rechtfertigen, deren Realisierung wirkt aber zusammen; vgl. hierzu die empirischen Befunde in Schmidt (2008, S. 367) und Nève (2009, S. 42). Dabei zeigt sich, dass in jenen Staaten, die einen hohen Grad an sozialstaatlicher Demokratie aufweisen, wie die skandinavischen Länder mit Ausnahme von Finnland, auch die Wahlbeteiligung am höchsten ausfällt. Hier soll jedoch nicht behauptet werden, dass eine hohe Inklusivität auf Seiten der demokratischen Verfahren eine höhere Sozialstaatlichkeit kausal bedingt. Vielmehr ist von einem Wechselverhältnis zwischen der Inklusivität der Beteiligung und dem Grad an sozialer Gerechtigkeit auszugehen. Bürger, denen es wirtschaftlich gut geht, gehen häufiger zur Wahl als jene, die sich von der Gesellschaft benachteiligt fühlen. Vor diesem Hintergrund erscheint es sowohl aus normativer als auch aus empirischer Sicht wenig überzeugend, das instrumentelle Versprechen der Demokratie auf Kosten des intrinsischen Wertes der politischen Gleichheit realisieren zu wollen. 
nen oder Bürgerversammlungen beteilige, ist umso unwahrscheinlicher, je geringer mein ökonomisches, mein kulturelles und mein soziales Kapital sind (Böhnke 2011; Geißel 2011). Dieser Befund ist nicht zuletzt zurückzuführen auf die Anspruchssteigerung, die mit den neuen bürgerschaftlichen Beteiligungsverfahren einhergeht. Der Prozess der Stimmabgabe ist vergleichsweise voraussetzungslos, das Engagement in Bürgerinitiativen oder die Teilnahme an Bürgerkonferenzen hängt jedoch von vielerlei Ressourcen ab. Neben der notwendigen Zeit sowie einem gewissen Maß an Sachkenntnis und Interesse, zählt hierzu der Glaube daran, etwas bewirken zu können - der Glaube an die eigene Fähigkeit zu handeln, und nicht zuletzt ein Selbstvertrauen, welches einem die nötige Kraft gibt, sich zusammen mit anderen zu engagieren. Diese Fähigkeiten sind jedoch in westlichen Demokratien ungleich verteilt. Minderprivilegierte Menschen verfügen weder über das notwendige soziale Kapital noch über das erforderliche Interesse, welches zum politischen Engagement motiviert.

„Die Fähigkeit zur Partizipation ist eben gebunden an besondere Ressourcen: Sprachgewandtheit, Kompetenz, Selbstbewusstsein, Informationen. Partizipation prämiert den privilegierten Zugang zu Bildungsgütern. Bürgergesellschaftliche Partizipation verfestigt und steigert so noch die Elitenstruktur moderner Demokratien; sie konsolidiert und fördert dadurch noch die Oligarchisierung des Willensbildungsprozesses“" (Walter 2009, S. 113).

Es spricht vieles dafür, dass die entsprechenden Partizipationsformen Lebensgewohnheiten, Ideale und auch Ressourcen voraussetzen, die bei sozial Benachteiligten nicht in einem ausreichenden Maße vorhanden sind. Besonders deliberative Arenen tendieren zur Exklusion bildungsferner und einkommensschwacher Menschen, und das in einem stärkeren Maße, als es bei den etablierten Verfahren der repräsentativen Demokratie der Fall ist. Aber auch direktdemokratische Verfahren weisen einen höheren sozialen Bias auf als etablierte Beteiligungskanäle (Schäfer u. Schoen 2013).

Zum anderen bieten die alternativen Beteiligungsverfahren die Möglichkeit für ressourcenstarke Bürger, Gesetzesvorhaben zu bekämpfen, die auf die Realisierung sozialer Chancengleichheit zielen, wie der Hamburger Volksentscheid zur geplanten Schulreform des damaligen schwarz-grünen Senates gezeigt hat. Ziel der Reform war es, Kinder aus bildungsfernen Milieus stärker zu fördern, unter anderem durch die Einführung einer sechsjährigen Primarschule. Gegen dieses Vorhaben sprachen sich die Hamburger Bürgerinnen und Bürger am 18. Juli 2010 mehrheitlich aus. Der Hamburger ,Schulstreit' hat von 1,3 Millionen Stimmberechtigten immerhin knapp 500.000 Bürgerinnen und Bürger zur Stimmabgabe motiviert. Doch war die Beteiligung ungleich verteilt; mobilisiert wurden vornehmlich die Bewohner der wohlhabenderen Stadtviertel, die der ,Problemviertel ${ }^{6}$ blieben demgegenüber größtenteils der Urne fern. Dieser Fall zeigt, dass ein Demokratieverständnis, welches die Widerspruchshandlungen der Bürger zum Kernbestandteil politischen Handelns erhebt, ohne auf die soziale Schieflage derartiger Verfahren zu achten, zumindest Gefahr läuft, das demokratische Versprechen der Gleichheit nicht nur in seiner politischen, sondern in der Konsequenz auch in seiner sozialen Dimension zu unterwandern. Direktdemokratische Verfahren sind 
zudem besonders anfällig für die Mobilisierung privater Vermögen zum Zwecke der Unterstützung oder auch Kontestierung politischer Vorhaben. Auch das konnte man beim Hamburger Volksentscheid gut beobachten. ${ }^{7}$

Die neuen Formen demokratischer Beteiligung, die immer auch als Instrumente einer ,Demokratisierung der Demokratie' angesehen worden sind und werden, haben also den gegenteiligen Effekt einer zunehmenden Oligarchisierung der Demokratie. ${ }^{8}$ Da dieser Sachverhalt sowohl aus einer demokratietheoretischen Perspektive, die sich der Norm der politischen Gleichheit verpflichtet weiß, als auch mit Blick auf das Ideal einer sozialen Demokratie unbefriedigend ist, möchte ich im folgenden Abschnitt alternative Verfahren demokratischer Beteiligung diskutieren, die explizit auf eine Stärkung minderprivilegierter Milieus zielen.

\section{Mehr Gleichheit durch deskriptive Repräsentation?}

Die Idee einer deskriptiven Repräsentation besagt, dass die Repräsentanten den Repräsentierten möglichst stark ähneln sollen, vor allem hinsichtlich ihrer sozialen, ökonomischen und auch ethnischen Eigenschaften. Legislative Kammern sollen ein Spiegelbild der in der jeweiligen politischen Gemeinschaft lebenden Bürgerinnen und Bürger darstellen. Auf diese Weise soll garantiert werden, dass sich die Repräsentanten nicht zu weit von der Lebenswelt und den Interessen der Repräsentierten entfernen, sie sich mithin responsiv gegenüber allen Bürgern und nicht nur gegenüber spezifischen Milieus verhalten. Verfechter der Idee einer deskriptiven Repräsentation kritisieren mithin, dass legislative Kammern, aber auch die Exekutive und Judikative, sich ohne entsprechende Vorkehrungen vornehmlich aus Angehörigen privilegierter Milieus zusammensetzen und in der Folge auch deren politische Perspektive einnehmen. ${ }^{9}$

Ohne Anspruch auf Vollständigkeit sollen im Folgenden drei Vorschläge aus der politikwissenschaftlichen Debatte der letzten Jahre diskutiert werden, die alle mehr politische Gleichheit durch eine Erhöhung der deskriptiven Repräsentation zum Ziel haben: Wahlpflicht (1), geloste Gremien (2) und Gruppenrechte (3). Ich

7 Volksinitiativen sind Decker (2011, S. 234) zufolge besonders anfällig für eine Instrumentalisierung durch „kampagnenstarke Minderheiteninteressen“, mit der Folge „einer systematischen Bevorzugung wirtschaftsnaher Produzenteninteressen, während soziale Anliegen oft das Nachsehen haben."

8 Das wird auch von Nolte anerkannt. Zwar distanziert er sich von „düsteren Visionen von der ,Postdemokratie““ (2012, S. 14) und er folgt in vielerlei Hinsicht der Einschätzung von Rosanvallon und Keane, doch am Ende seines Buches finden sich skeptische Töne: „Die neue Demokratie ist die einer mehr individualistischen, nicht mehr kollektiv organisierten Gesellschaft. Davon profitieren diejenigen sozialen Schichten, die den Individualismus und die neuen Netzwerkstrukturen zur Grundlage ihres Lebens und ihres beruflichen Erfolges machen konnten: die Mittelklassen“ (2012, S. 437-438).

9 Zum Modell der ,descriptive representation' vgl. Pitkin (1967, S. 60-91), im Anschluss an Pitkin und mit Blick auf die Vorstellungen der Anti-Federalists auch Manin (2007, S. 152-182). Die klassische Formulierung dieses Konzepts stammt von John Adams: "As the representative assembly should be an exact portrait, in miniature, of the people at large, as it should think, feel, reason, and act like them, great care should be taken in the formation of it, to prevent unfair, partial, and corrupt elections" (Adams 1776, S. 205). 
werde mich gegen die Einführung einer Wahlpflicht aussprechen und auch den anderen beiden Reformvorschlägen nur bedingt folgen.

\subsection{Deskriptive Repräsentation durch Wahlpflicht}

Um dem sozialen Bias von Wahlen zu begegnen, hat Armin Schäfer jüngst die Einführung einer Wahlpflicht vorgeschlagen. ${ }^{10}$ Sein empirisch gestütztes Argument hierfür lautet zunächst, dass der Rückgang der Wahlbeteiligung sich nicht gleichmäßig auf alle sozialen Milieus verteilt, sondern vor allem minderprivilegierte Bürger betrifft. Sodann zeigt er, dass eine niedrige Wahlbeteiligung mit der Gefahr einhergeht, dass die Interessen der sozial Schwachen vernachlässigt werden: "If certain groups abstain from voting, politicians may ignore their interests and instead appease those who are likely to vote" (2011, S. 9). Tatsächlich deuten die von Schäfer zitierten empirischen Studien darauf hin, dass eine hohe Wahlbeteiligung zu höheren Wohlfahrtsausgaben führt und dass in Staaten mit Wahlpflicht die Einkommensunterschiede weniger stark ausgeprägt sind (S. 12-13).

Das sind insbesondere vor dem Hintergrund des normativen Ziels einer sozialen Demokratie gewichtige Argumente. Dennoch sprechen zwei Gründe gegen die Einführung einer Wahlpflicht. Erstens ist diese nur dort effektiv, wo die Nichtbefolgung sanktioniert wird, in der Regel mit Bußgeldern, bei der Weigerung diese zu zahlen auch mit Gefängnisstrafen. Gerade sozial schwache Bürger würden derartige Strafen belasten. Sollten sie zu einer entsprechenden Zahlung verpflichtet werden, wird das sicherlich nicht zur Systemzufriedenheit beitragen. Doch auch im positiven Fall einer Teilnahme ist fraglich, ob eine Bußgelddrohung zu jener erhabenen Stimmung beiträgt, die viele Menschen mit dem Akt der Wahl verbinden. Zu befürchten ist vielmehr eine Art Antistimmung, wenn man zu etwas gezwungen wird, was man eigentlich - aus welchen Gründen auch immer - ablehnt. Das könnte bestenfalls zu einer Erhöhung ungültiger Wahlstimmen, aber auch zu Gewinnen demokratiefeindlicher Parteien führen.

Zweitens sind demokratische Beteiligungsformen im Allgemeinen und Wahlen im Speziellen immer auch Rituale, in denen die symbolische Integration der Gesellschaft sichergestellt und reproduziert wird. Sie dienen ebenso der Vergewisserung eines Zusammengehörigkeitsgefühls wie der Bestätigung fundamentaler politischer Ideale. Andreas Dörner spricht hier von dem demokratischen Mythos, durch dessen Inszenierung das demokratische Imaginäre immer wieder aufs Neue aktualisiert und reproduziert wird:

„Daher kommt auch dem Wahlkampf als Ritual eine so zentrale Bedeutung zu: Er verleiht dem demokratischen Mythos besondere Überzeugungskraft. [...] Rituale geben Orientierungssicherheit, und sie vermitteln den Menschen das Gefühl, bei den wirklich wichtigen Dingen ihrer Bezugsgemeinschaft ,dabeizusein““ (Dörner 2002, S. 29).

Die Bedeutsamkeit demokratischer Wahlen sowie unkonventioneller Formen politischen Engagements besteht also zumindest auch in der wechselseitigen Verge-

10 Eine Wahlpflicht fordern auch Merkel u. Petring (2011). 
wisserung, ein ,guter Demokrat' zu sein. In dieser Bezugsgemeinschaft können sich viele Menschen aber kaum wiederfinden und der Gang zur Wahl würde aus ihrer Sicht eine Ordnung legitimieren, die sie als ungerecht erfahren. Warum sollten sie dann ein Ritual befolgen, von dem sie sich nichts (mehr) erhoffen können? Warum zur Wahl gehen, wenn das sowieso wenig bis nichts an der persönlichen Situation ändert? Der Akt des Wahlboykotts stellt somit auch einen moralischen Appell an die Mehrheitsgesellschaft und ihre Meinungsführer dar, das, was sie alle vier Jahre zelebrieren, nicht nur in seinem symbolischen Gehalt beim Wort zu nehmen. Eine Wahlpflicht mit entsprechenden Sanktionen würde diese Möglichkeit des Ausdruckes einer grundlegenden Unzufriedenheit verbauen und es ist fraglich, ob eine Erhöhung des Anteils der ungültigen Stimmen den gleichen Effekt besitzen würde.

\subsection{Deskriptive Repräsentation durch Losverfahren}

Auch Losverfahren zielen auf eine möglichst deskriptive Repräsentation und haben in jüngster Zeit geradezu einen Boom in der Literatur erlebt (vgl. u. a. Buchstein 2009; Stone 2010). Die Idee, durch Losen politische Gremien zu bestimmen, kann in der Praxis nicht nur auf antike und frühneuzeitliche Vorläufer zurückblicken, sondern ist auch in gegenwärtigen Demokratien im Rahmen von ,deliberative opinion polls' zum Einsatz gekommen, bei denen per Zufall ausgewählte Bürger politische Probleme diskutieren sollen. Diesen und ähnlichen zufallsgenerierten Gremien wird in der Literatur insbesondere zugeschrieben, dass sie keinen sozialen Bias aufweisen und gerade deshalb zu einer unparteilichen Beurteilung gelangen können (vgl. Buchstein 2009, S. 383-384). Allerdings besitzen die ausgelosten Versammlungen oftmals keine politischen Entscheidungsrechte, also keinen Einfluss, ihnen wird lediglich zugeschrieben, den Wissensstand und die politische Urteilskraft der beteiligten Bürger zu erhöhen (Fishkin 1991) oder ihnen wird eine konsultative Funktion im Prozess der Gesetzgebung zugewiesen (Dahl 1989).

Weiter gehen Hubertus Buchstein und Michael Hein (2009) in ihren Überlegungen zum Einbau von Losverfahren in das Institutionengefüge der Europäischen Union. Konkret schlagen sie neben der Auslosung der Kommissare und der Ausschussvorsitzenden des Europäischen Parlaments ein ,House of Lots' als zweite Kammer neben dem Europäischen Parlament vor, die aus 200 Mitgliedern besteht, welche alle zweieinhalb Jahre aus den wahlberechtigten Einwohnern der 27 Mitgliedsländer ausgelost werden. Das ,House of Lots` soll nicht nur Empfehlungen aussprechen dürfen, sondern besitzt darüber hinaus auch ein Initiativrecht sowie „ein absolutes Vetorecht“ gegen Legislativakte (Buchstein u. Hein 2009, S. 373). Der Reiz dieses Vorschlages besteht darin, dass nicht Vertreter von Parteien oder NGOs, sondern eine statistisch repräsentative Auswahl der europäischen Bürgerschaft auf diese Weise zu einer wichtigen Stimme im Gesetzgebungsverfahren erhoben wird. Eine derartige Loskammer hätte also durchaus politischen Einfluss im europäischen Mehrebenensystem.

Ausgeloste Versammlungen, sofern garantiert ist, dass auch alle gelosten Bürger partizipieren und die Größe des Gremiums zufallsbedingte Verzerrungen weitgehend ausschließt, erhöhen die deskriptive Repräsentation und können somit zu 
einer stärkeren Berücksichtigung der Perspektiven minderprivilegierter Milieus beitragen. Inwieweit das tatsächlich der Fall ist und inwieweit diese Perspektiven dann auch politisch effektiv werden, hängt jedoch von dem jeweiligen Design und den jeweiligen Rechten ab. Die Vorschläge von Buchstein und Hein sind hier die am weitestgehenden und vielversprechendsten. Allerdings trifft, wenn auch in abgeschwächter Weise, auf diese Losgremien ebenfalls zu, was oben mit Blick auf deliberative Versammlungen beschrieben wurde. Denn auch hier dürften ressourcenstarke Bürger größere Chancen haben, sich Gehör und in der Konsequenz auch Einfluss zu verschaffen. Diesem Effekt lässt sich gewiss begegnen, etwa durch eine entsprechende Moderation oder durch die Zuteilung von Redezeiten oder anderen Ressourcen, gänzlich aufheben lässt er sich nicht (vgl. Schaal u. Ritzi 2012, S. 141).

\subsection{Deskriptive Repräsentation durch Gruppenrechte}

Die Idee der deskriptiven Repräsentation ist nicht zuletzt von feministischen Autorinnen aufgegriffen worden, die eine mangelnde Vertretung von Frauen in politischen Institutionen, aber auch in der Öffentlichkeit kritisieren. Bereits Anfang der 1990er-Jahre hat Iris M. Young (1990) darauf aufmerksam gemacht, dass politische Entscheidungsverfahren, für die Gruppendifferenzen irrelevant sind, dazu tendieren, bestimmte ökonomisch und kulturell bedingte Formen der Unterdrückung zu verfestigen, da benachteiligte Gruppen im politischen Prozess nur über eine schwache Stimme verfügen. Moderne Demokratien nehmen zwar Neutralität für sich in Anspruch, tatsächlich werde jedoch eine partikulare Perspektive privilegiert, nämlich diejenige gebildeter, weißer Männer. Konkret hieße das, dass etwa im öffentlichen Diskurs spezifische Regeln hinsichtlich dessen gelten, was als Argument zählt und auf welche Weise es vorgebracht werden darf, aber auch dahingehend, wer als geeigneter Kandidat bei einer Wahl angesehen wird und wer nicht. Im Ergebnis führe dies dazu, dass auch in der politischen Öffentlichkeit sowie im Prozess der Gesetzgebung die Perspektive und die Interessen der dominanten Gruppen vorherrschen, unterdrückte Gruppen jedoch nur am Rande Gehör fänden, geschweige denn über politischen Einfluss verfügen.

Um den diskriminierenden Effekten formal neutraler Verfahren demokratischer Entscheidungsfindung zu begegnen, schlägt Young Mechanismen einer , politics of difference" vor. "(A) democratic public should provide mechanisms for the effective recognition and representation of the distinct voices and perspectives of those of its constituent groups that are oppressed or disadvantaged" (Young 1990, S. 184). Zu diesen Mechanismen gehören Vetorechte, Quotenregelungen und die finanzielle Förderung der Selbstorganisation. Young ist der Auffassung, dass diese Mechanismen die Repräsentation der Interessen benachteiligter sozialer Gruppen in der Öffentlichkeit und im Prozess der kollektiven Entscheidungsfindung stärken können. Ihr zufolge bewirke deren spezifische Förderung jedoch nicht nur mehr politische Gleichheit. Darüber hinaus würde dadurch auch eine Pluralisierung der Perspektiven erfolgen, welche die „practical wisdom“ (Young 1990, S. 186) von kollektiven Entscheidungen erhöht. Der Einbezug verschiedener Ge- 
sichtspunkte in den Prozess der politischen Willensbildung führe zu mehr Wissen und weniger Ungerechtigkeit. ${ }^{11}$

Die Vorschläge von Young sind nicht unwidersprochen geblieben. Namentlich Anne Phillips hat auf mehrere Probleme hingewiesen. An erster Stelle ist das der Festlegung der soziokulturell benachteiligten Gruppen zu nennen: "Do we say, for example, that wherever a group feels itself oppressed or discriminated against, then there is a prima facie case for guaranteeing that group some form of representation?" (Phillips 1993, S. 97, Herv. i. O.). Auch müsse das Prinzip der Gruppenrepräsentation nicht unbedingt die Interessen sämtlicher Mitglieder der repräsentierten Gruppe adäquat widerspiegeln. So könnten gerade durch die Mechanismen einer besonderen Förderung, etwa durch finanzielle Ressourcen oder einen privilegierten Zugang zu staatlich finanzierten Medien, neue Oligarchien innerhalb der Gruppe entstehen, die dann zwar beanspruchen, für alle Gruppenmitglieder zu sprechen, letztlich aber doch nur ihre partikularen Interessen verfolgen. Zudem bestehe die Gefahr der Verfestigung von Gruppenegoismen durch eine zu starre Institutionalisierung der Gruppenvertretung. Und schließlich macht Phillips darauf aufmerksam, dass auch benachteiligte Gruppen nicht a priori im Recht sind: "The oppressed have no monopoly on good behaviour; being a victim is not a guarantee of right" (Phillips 1993, S. 160).

Die Einwände von Phillips sind gewichtig. ${ }^{12}$ Dennoch sollten sie nicht dazu verführen, die Idee einer spezifischen Gruppenrepräsentation gerade auch mit Blick auf sozial benachteiligte Milieus vorschnell zu verabschieden. So ist es relativ einfach, deren Angehörige zu bestimmen, etwa die Bezieher von Sozialtransferleistungen oder jene, deren Einkommenssteuer eine bestimmte Höhe nicht überschreitet. Ebenso kann keine Rede davon sein, dass sozial benachteiligten Bevölkerungsgruppen immer und notwendig eine normativ höher zu bewertende Perspektive zukommt. Es geht auf einer demokratietheoretischen Ebene nicht um die normative Beurteilung der jeweiligen Interessen und Werte, sondern darum, die entsprechenden Perspektiven so in den politischen Prozess einzuspeisen, dass sie überhaupt Zugang und Gehör finden, sie dort dann aber auch - durch Mehrheitsentscheid - zurückgewiesen werden können. Insofern wir davon ausgehen müssen, dass in Verfahren, zu denen formal alle sozialen Milieus Zugang besitzen, sozial Benachteiligte nicht, oder nur marginal repräsentiert sind, und wir deren mangelnde Repräsentation aus normativen Gründen überwinden wollen, bieten milieuspezifische Beteiligungsrechte daher zumindest eine Option an, dem Problem der ungleichen Beteiligungsintensität zu begegnen. Dabei kommt es aber, und das hat die Kritik von Phillips verdeutlicht, auf die konkrete Form der Umsetzung an.

Young selbst hat sich in einem späteren Aufsatz (1997) von der Forderung nach einer besonderen Gruppenrepräsentation in Parlamenten und dem damit

11 Eine aktuelle Verteidigung des epistemischen Werts von Differenz liefert Landemore (2013).

12 Die Vorschläge von Young haben eine breite Diskussion ausgelöst. Neben den hier im Anschluss an Phillips vorgebrachten Einwänden wurde aus differenztheoretischer Perspektive insbesondere vor vermeintlich ,essentialistischen` Implikationen gewarnt. Demgegenüber hat Young zu Recht den ,fluiden` und konstruierten Charakter sozialer Gruppen betont. Für einen aktuellen Überblick über die Debatte vgl. Thaa (2013). 
verbundenen Ideal einer deskriptiven Repräsentation distanziert. Sie begreift nun Repräsentation als eine Beziehung, die politische Identitäten gerade erst konstituiert. Insbesondere hat sie sich von der Forderung nach Vetorechten verabschiedet. Waren mit Vetorechten auch Entscheidungsbefugnisse verbunden, so lehnt sich Young in „Inclusion and Democracy“ (2000) stärker an die deliberative Demokratietheorie an und betont die Notwendigkeit der Einspeisung möglichst aller Perspektiven in den politischen Beratungsprozess. Allerdings geht dies, wie Winfried Thaa (2009. S. 72-73) betont, mit einer Vernachlässigung des Ungleichheitsproblems einher. Das zeigen auch die Erfahrungen, die bislang mit der Umsetzung von Gruppenrechten in etablierten Demokratien gemacht worden sind.

Denn die von Young vorgeschlagenen Mechanismen zur Einspeisung verschiedener Perspektiven sind in westlichen Demokratien bereits verwirklicht. Dazu zählen vor allem Quotenregelungen, die etwa zu einer Erhöhung des Frauenanteils oder von Vertretern nationaler Minderheiten in Parteigremien und Parlamenten geführt haben und damit auch zu einer stärkeren Berücksichtigung ihrer Perspektiven geführt haben (vgl. Knaut 2011; Geißel 2012). Ähnliche Quotenregelungen, die den Anteil sozial Benachteiligter in Parlamenten erhöhen sollen, sind aber weder vorhanden noch sinnvoll, und zwar auch aus der hier diskutierten Perspektive des Ideals einer sozialen Demokratie. Einmal abgesehen davon, dass zusätzliche Quotenregelungen zu einer erheblichen Einschränkung der Wahlfreiheit führen würden, ist insbesondere fraglich, ob damit tatsächlich das Ziel einer stärkeren Repräsentation schwacher Interessen erreicht werden könnte. So würde etwa mit der Übernahme eines Abgeordnetenmandats und der sodann nahezu zwangsläufig einsetzenden Professionalisierung auch die Zugehörigkeit zum Milieu der sozial Benachteiligten enden. Einsetzen dürften jene Prozesse der, Verbürgerlichung', die Robert Michels (1989) in seiner „Soziologie des Parteiwesens“ bereits vor 100 Jahren mit Blick auf die Funktionsträger der SPD beschrieben hat. ${ }^{13}$

Auch die von Young vorgeschlagene Stärkung der Interessenvertretung benachteiligter Gruppen durch Förderung ihrer Selbstorganisation und deren Berücksichtigung im Gesetzgebungsprozess ist zumindest teilweise längst Realität. So werden Frauenverbände, Migrationsverbände und auch Arbeitslosenverbände vom deutschen Staat unterstützt und regelmäßig zu parlamentarischen Anhörungen und runden Tischen eingeladen. Die jeweiligen Perspektiven finden im politischen Prozess also durchaus Gehör. Eine andere Frage ist freilich die nach dem Einfluss dieser Verbände, der als eher gering eingeschätzt werden muss (vgl. Linden u. Thaa 2009). Hinzu kommen hier ebenfalls der Prozess der Professionalisierung und die damit verbundene Gefahr einer Verselbstständigung der Verbandseliten.

Kurzum, um eine stärkere Berücksichtigung der Interessen sozial Benachteiligter im politischen Prozess zu erreichen, sind sowohl Quotenregelungen als auch klassische Formen des Lobbyismus nur bedingt zielführend. Anders könnte es bei gruppenspezifischen Vetorechten aussehen. Mit Blick auf sozial Benachteiligte werde ich daher gegen Young an der Notwendigkeit auch von milieuspezifischen

13 Dass das von Michels beschriebene Repräsentationsdefizit auch zu Beginn des 21. Jahrhunderts besteht, zeigt Borchert (2003). 
Entscheidungsrechten festhalten. Diese stellen allerdings auf der prozeduralen Ebene einen Bruch mit der demokratischen Gleichheitsprämisse dar. Bevor ich daher einen Vorschlag skizziere, der Veto-Rechte für soziale Benachteiligte vorsieht, soll zunächst dieser Schritt begründet werden.

\section{Mehr politische und soziale Gleichheit durch ungleiche Beteiligungsrechte}

\subsection{Die exkludierenden Effekte der Gleichheitsfiktion}

Für moderne Demokratien ist die Norm der politischen Gleichheit grundlegend. Auch wenn sich diese Norm nach der Französischen Revolution erst sukzessive durchgesetzt hat und in den ideengeschichtlichen Debatten zunächst gerade nicht für ein universelles Wahlrecht plädiert wurde, so ist damals doch der Keim der Vorstellung entstanden, dass politische Macht weder bei einem König oder einer Aristokratie noch bei Experten, weder bei den Protestanten noch bei den Katholiken, weder bei den Männern noch bei den Frauen, weder bei den Akademikern noch bei den Arbeitern konzentriert sein darf. Vielmehr soll jede Stimme gleich viel zählen. Für Robert A. Dahl ist dieses „strong principle of equality“ wie folgt charakterisiert:

"The members [of a political community, DJ] believe that no single member, and no minority of members, is so definitely better qualified to rule that the one or the few should be permitted to rule over the entire association. They believe, on the contrary, that all the members of the association are adequately qualified to participate on an equal footing with the others in the process of governing the association" $(1989$, S. 31).

Seit einiger Zeit werden in der politikwissenschaftlichen Literatur jedoch Zweifel am demokratischen Charakter von Wahlen artikuliert. Im vorigen Abschnitt habe ich bereits die einflussreiche Kritik von Young an formaldemokratischen Verfahren skizziert. Die selektiven Wirkungen der etablierten demokratischen Institutionen werden auch von Bernard Manin beklagt. In seiner ideengeschichtlichen Rekonstruktion der repräsentativen Regierungsform (2007) argumentiert er zunächst, dass dem Wahlverfahren bis in die frühe Neuzeit ein aristokratischer Charakter zugeschrieben wurde, wobei er sich auf Aristoteles, Montesquieu und Rousseau beruft. Für diese zählten Wahlen insofern zu einem aristokratischen System, als durch sie die ,Besten' ausgewählt würden. Das genuin demokratische Bestellungsverfahren sei dagegen das Losprinzip. Manin erwähnt zwar, dass Wahlen in der Moderne sowohl „den aristokratischen Sinn einer Heraushebung der Besseren und des Führers oder aber den demokratischen Sinn der Bestellung eines Agenten, Kommissars oder Dieners“ (2007, S. 207) haben können, doch er zeigt ebenso auf, dass es vor allem der aristokratische Charakter des Wahlsystems war, welcher dieses für einflussreiche politische Denker wie die Autoren der „Federalist Papers" so attraktiv gemacht hat. Für Manin ist dieser aristokratische Charakter trotz aller Wahlrechtserweiterungen auch im Zeitalter der repräsentativen Demokratie weiterhin wirksam, wobei er insbesondere die Unabhängigkeit der Repräsentanten sowie die Tatsache, dass Wahlen im Unterschied zum Losen nicht jedem 
die gleiche Chance einräumen, ein politisches Amt auszuüben, als einen aristokratischen Bestandteil westlicher Demokratien ansieht. Dies führt ihn am Ende seines Buches zu dem Urteil, dass repräsentative Demokratien besser als Mischverfassungen zu qualifizieren seien (2007, S. 326). ${ }^{14}$

Im Anschluss an Manin hat John McCormick (2011) ebenfalls die Gleichheit des Wahlverfahrens bestritten. Auch für ihn besitzen Wahlen einen „aristocratic effect“", der darin besteht, dass Reiche und Prominente „enjoy a persistent advantage in generally wide electoral contests" (2011, S. 98). Für McCormick ist vor allem die Entwicklung in den USA besorgniserregend, da dort die Wohlhabenden den politischen Prozess zunehmend kontrollierten. Der Grund hierfür bestehe darin, dass politische Institutionen, die prinzipiell für alle zugänglich sind, unter sozialen Bedingungen, in denen einige wenige über erhebliche Ressourcenvorteile verfügen, von diesen okkupiert oder zumindest dominiert werden. ${ }^{15}$ Anders ausgedrückt: Das normatives Ideal, nämlich das der politischen Gleichheit, hat McCormick zufolge zu einer Institutionalisierung geführt, die dessen Gehalt in der Realität geradezu entgegengesetzt ist. Ähnlich wie Young sieht er die entscheidende Ursache für diese Entwicklung in einer demokratietheoretischen Gleichheitsfiktion, die sich am augenfälligsten in dem Grundsatz ,one (wo)man, one vote' niederschlägt. Das allgemeine Wahlrecht und die aus den Wahlen hervorgehenden Parlamente und Regierungen würden, so die demokratietheoretische Grundannahme, allen Bürgern den gleichen Anteil am Prozess der Selbstgesetzgebung bzw. der Selbstregierung zubilligen. Doch diese Annahme ist für McCormick nicht nur falsch, sie hat darüber hinaus auch eine ideologische Wirkung. Denn die Politikergebnisse würden auf diese Weise gegen die Kritik aus einer der politischen Gleichheit verpflichteten Perspektive immunisiert. Ein wesentliches Problem der gegenwärtigen Demokratien bestehe also darin, dass sie von einer Gleichheitsfiktion ausgehen, die die exkludierenden Effekte der existierenden Verfahren und Praktiken verschleiert. Eben darin unterscheiden sie sich von früheren Modellen einer Mischverfassung:

"As a result of class-anonymity and sociological 'holism', modern constitutions seem less concerned with, and may be less adept at, keeping wealthy citizens from dominating politics than were traditional constitutions in

14 Manin sieht aristokratische Tendenzen insbesondere in der „Publikumsdemokratie“, die in den 80er-Jahren des 20. Jahrhunderts in westlichen Gesellschaften entstanden ist. Dem vorherigen Typus der „Parteiendemokratie“ bescheinigt er demgegenüber einen vergleichsweisen hohen demokratischen Charakter.

15 Diese Einschätzung ist jüngst von Jeffrey Winters empirisch bestätigt worden. Winters bezeichnet die USA daher auch trotz des allgemeinen Wahlrechts als eine Oligarchie und stellt sie auf eine Ebene mit den Philippinen. Beide politische Systeme besitzen zwar demokratische Institutionen, doch diese können von den wenigen Superreichen umgangen werden: "It is clear that oligarchy coexists remarkably easily with democracy. This means that achieving democracy, especially if this only means implanting the democratic method, is not a solution to the oligarchy problem. There is nothing automatic about ending oligarchy through the adoption of free and participatory forms of government" (Winters 2011, S. 284). Von derartigen Verhältnissen mögen die westeuropäischen Demokratien (noch) entfernt sein. In der Öffentlichkeit hat sich jedoch das Bild verfestigt, dass die Maßnahmen zur „Eurorettung“ vornehmlich den Interessen der Kapitalbesitzer dienen, laut Streeck (2013) durchaus nicht unbegründet. 
which the people comprised a subset, the largest subset, of the citizenry" (2011, S. 12).

McCormick schlägt nun vor, auch in modernen Demokratien wieder klassensensitive Institutionen einzuführen, wobei er sich auf eine Rekonstruktion von Machiavellis Überlegungen zur Mischverfassung stützt. Machiavelli ging insbesondere in seinen Discorsi mit Blick auf die Römische Republik und auf die politische Ordnung von Florenz zu seiner Zeit von einem unaufhebbaren Interessengegensatz zwischen den grandi und den popolo aus und argumentierte, dass nur ein System, in dem die Plebejer über eigene Institutionen - etwa das Volkstribunat oder eigene Versammlungen - verfügen, diese vor der Beherrschung durch die grandi schützt. Mit Machiavelli skizziert McCormick für die heutigen USA ein institutionelles Design, welches soziale Unterschiede und die damit verbundenen ungleichen Ressourcen anerkennt und eben dadurch aristokratische Tendenzen vermeiden soll. Konkret plädiert er für die Einrichtung einer Tribunatsversammlung als eine Art Zweite Kammer, welche sich aus jährlich ausgelosten Bürgerinnen und Bürgern zusammensetzen soll. ${ }^{16}$ Laut McCormick soll es sich dabei insofern um eine klassenspezifische Kammer handeln, als politische Eliten und besonders wohlhabende Bürger dort nicht vertreten sein dürfen. Das Ziel ist, durch Losen eine Kammer zu besetzen, in der der ,common sense' der Bevölkerung als Gegengewicht zu den professionalisierten Sicht- und Denkweisen politischer Eliten vertreten ist. Die Aufgabe der auf diese Weise zusammengesetzten Tribunatsversammlung besteht in der Überwachung der Gesetzgebung und des Regierungshandelns. McCormick weist den Tribunen mit Blick auf das politische System der USA Vetorechte gegen Legislativakte zu, das Recht ein ImpeachmentVerfahren zu eröffnen und das Recht, ein Referendum zu initiieren. Gerade Letzteres ist geeignet, Themen, die von den politischen Eliten entweder nicht verfolgt werden, oder die sie aktiv bekämpfen, auf die politische Agenda zu setzen. Dies kann auch Maßnahmen zur Verminderung sozialer Ungleichheit oder zur Begrenzung sozialer Macht beinhalten. Allerdings können Referenden, wie der Hamburger Schulstreit gezeigt hat, auch zu gegenteiligen Effekten führen.

\subsection{Ein Verfahrensvorschlag: Vetorechte für sozial Benachteiligte}

Politische Verfahren, die allgemein zugänglich sind, tendieren dazu, gesellschaftliche Ungleichheiten zu reproduzieren. Dieser Befund gilt nicht nur für Wahlen, sondern in verstärkter Weise auch für alternative Beteiligungsverfahren. Auch die oben diskutierten Maßnahmen zur Realisierung der deskriptiven Repräsentation können der Tendenz zur Privilegierung ressourcenstarker Bürger nur bedingt entgegenwirken. Insofern stellen alternative Beteiligungsverfahren, die formal allgemein zugänglich sind, keinen Ausweg aus der postdemokratischen Konstellation dar. Um diesem Sachverhalt institutionell zu begegnen, möchte ich den Gedanken von McCormick, mehr politische Gleichheit durch ungleiche Beteiligungsrechte

16 Die jährliche Auslosung soll einer Professionalisierung der Tribunen entgegenwirken. 
zu erzielen, aufgreifen und mit der ursprünglichen Idee von Young kombinieren, Vetorechte für benachteiligte Gruppen einzuführen.

Mein Vorschlag besteht darin, minderprivilegierten Milieus ein Vetorecht im Rahmen eines fakultativen Referendums einzuräumen. Es sollte sich dabei nicht um ein absolutes, sondern lediglich um ein aufschiebendes Veto handeln, das zudem auf Gesetzesvorhaben im Bereich der Bildungs,- Sozial- und Wirtschaftspolitik beschränkt sein sollte - wie etwa die ,Hartz-Reformen' in der Bundesrepublik Deutschland oder auch die gegenwärtigen ,Konsolidierungsprogramme“ in Spanien, Portugal, Italien und Griechenland. ${ }^{17}$ Im Unterschied zu klassischen Referenden und auch zu Wahlverfahren dürfen sich nicht alle Bürgerinnen und Bürger an diesen Verfahren beteiligen, sondern lediglich sozial Benachteiligte. Teilnahmeberechtigt sind also lediglich diejenigen Bevölkerungskreise, die am demokratischen Prozess deutlich unterproportional partizipieren. Das Ziel dieses Vorschlages besteht darin, den Angehörigen minderprivilegierter Milieus zumindest mehr Gehör, jedoch nicht unbedingt mehr Einfluss zu verschaffen. ${ }^{18}$ Es soll dazu beitragen, deren Interessen und Bedürfnisse wieder stärker in jenen politischen Entscheidungen zur Geltung zu bringen, die die grundlegende Verteilung von Gütern und Lebenschancen betreffen. ${ }^{19}$

Auch für Heidrun Abromeit (2002, S. 177-190) sind fakultative Referenden als Veto-Instrumente in besonderer Weise geeignet, die Interessen jener Minderheiten zu schützen, die gewöhnlich überstimmt werden. Das gilt ihr zufolge vor allem in jenen Gesellschaften, die durch große kulturelle und soziale Differenzen charakterisiert sind. Abromeit (2002, S. 180) hebt jedoch zu Recht hervor, dass die auslösende Instanz das „wichtigste Kriterium“ zur Beurteilung direktdemokratischer Verfahren darstellt. Mit Blick auf das hier vorgeschlagene Modell, das Beteiligungsrechte spezifisch für ressourcenschwache Bürger vorsieht, stellt sich dieses Problem in besonderer Schärfe, da diese Bevölkerungsgruppen auf der einen Seite gemeinhin nicht über das erforderliche Maß an politischen Engagement verfügen. Es ist also schwer vorstellbar, dass ein entsprechendes Volksbegehren ,bottom up

17 Die Beschränkung auf diese Policy-Bereiche ergibt sich aus dem Umstand, dass hier wesentliche Ressourcen und Zugangschancen verteilt werden.

18 Ein suspensives Vetorecht würde auf jeden Fall dazu beitragen, dass minderprivilegierte Bürger mehr Gehör bekommen. Mehr Einfluss verspräche dagegen ein absolutes Vetorecht. Dieses könnte dann aber zu erheblichen Politikblockaden führen.

19 Ähnlich auch die Argumentation von Linden u. Thaa (2011, S. 314): „Zu diskutieren wäre demzufolge, ob fakultative Referenden, also die Kontestierung eines parlamentarisch beschlossenen Gesetzes durch eine Vetoinitiative der Bürger, nicht die Möglichkeit bieten, einen über Mehrheitsinteressen oder -meinungen hinweggehenden Elitenkonsens aufzubrechen und die vermeintlich unumgänglichen Erfordernisse, mit denen Entscheidungen allzu gern begründet werden, politisch zur Disposition zu stellen." Mein Vorschlag teilt deren Zielrichtung, geht aber insofern darüber hinaus, als der Kreis der Teilnahmeberechtigten auf minderprivilegierte Bürgerinnen und Bürger beschränkt werden soll. 
erfolgt. Auf der anderen Seite sind fakultative Referenden anfällig für parteipolitische und insbesondere populistische Instrumentalisierungen. ${ }^{20}$

Diesem doppelten Problem könnte die oben vorgestellte Tribunatsversammlung begegnen. Um ein Referendum zu initiieren, ist McCormick zufolge eine einfache Mehrheit der Tribunen erforderlich. Ist dieser Schritt erfolgt, dann ist entscheidend, im Referendumsprozess den Einfluss von Interessengruppen und politischen Parteien so weit wie möglich zu begrenzen:

"The referendum will take place without any advertising sponsored by parties or interest groups. A nationally televised debate between two tribunate approved advocates - policy experts, public officials, or private citizens one in favor of and one opposed to the proposition, will precede the referendum" (2011, S. 184).

Allein die Drohkulisse eines derartigen Referendums könnte die politischen Eliten dazu bewegen, die Interessen und Bedürfnisse jener Menschen, die über wenig soziales Kapital verfügen, stärker zu berücksichtigen, als es in den vergangenen 20 Jahren der Fall gewesen ist. Damit soll für sozial benachteiligte Milieus jene negative Allgemeinheit gewährt werden, die Rosanvallon in den Verfahren der ,Counter-Democracy' realisiert sieht. Allerdings haben wir gesehen, dass aufgrund der sozialen Selektivität alternativer Beteiligungsverfahren diese Vetomacht vornehmlich den gut ausgebildeten Mittelschichten zukommt. Es geht mit meinem Vorschlag also darum, auch für ressourcenschwache Menschen Wege der Kontestierung politischer Entscheidungen zu eröffnen. Dabei gibt es natürlich keine Garantie, dass entsprechende Referenden sich nicht auch gegen Maßnahmen zur Bekämpfung sozialer Ungleichheit richten könnten. Die Hoffnung ist jedoch, dass entsprechende Gruppenrechte zumindest der oben erwähnten Passivierung politischer Beteiligung im Rahmen der postdemokratischen Konstellation entgegenwirken könnten.

Neben verfassungsrechtlichen Einwänden, denen ich im abschließenden Abschnitt die Idee einer experimentellen Demokratie entgegenstelle, könnte an dieser Stelle ein eher pragmatisches Argument gegen die Einführung milieuspezifischer Vetorechte vorgebracht werden. So ließe sich argumentieren, dass durch zusätzliche Vetospieler die Wahrscheinlichkeit einer Politikblockade erhöht werde. Diesem Einwand wird hier insofern entsprochen, als es sich erstens nur um ein suspensives Veto handeln soll, zweitens das Instrument auf wesentliche Reformen in jenen Politikfeldern beschränkt ist, die Auswirkungen auf die Verteilung von grundlegenden Gütern und Chancen haben, und drittens mit dem Modell der Tribunatsversammlung eine zusätzliche Hürde verbunden ist.

20 Decker (2011, S. 235) argumentiert, dass direktdemokratische Elemente nicht, wie man vielleicht mit Verweis auf das Minarettverbot in der Schweiz annehmen könnte, notwendig den Effekt einer Steigerung populistischer Tendenzen haben müssen. Dagegen spricht zum einen, dass „populistische Positionen [...] offen auf den Tisch gelegt werden müssen“ und infolge einer gesellschaftlichen Diskussion „Stimmungen rasch abkühlen“ können. Zum anderen können Referenden auch eine „Ventilfunktion“ haben, was insbesondere dann wichtig ist, wenn die politischen Eliten „zur Abgehobenheit neigen und nicht alle Interessen gleichberechtigt Zugang zum Entscheidungsprozess haben.“ 


\section{Fazit: Die Notwendigkeit eines demokratischen Experimentalismus}

Der im vorigen Abschnitt formulierte Vorschlag eines fakultativen Referendums, an dem sich nur sozial Benachteiligte beteiligen dürfen, ist im Sinne eines demokratischen Experimentalismus zu verstehen, wie er von John Dewey in „Die Öffentlichkeit und ihre Probleme" eingefordert worden ist. Politische Verfahren besitzen für Dewey lediglich einen vorläufigen Status und auch institutionelle Normierungen der demokratischen Meinungs- und Willensbildung haben nur so lange ihre Berechtigung, wie sie ihren Zweck erfüllen. Das kann sich aber im Laufe der Zeit verändern. Bestimmte Institutionen wie etwa das allgemeine Wahlrecht, repräsentative Regierungsform und Mehrheitsprinzip haben für ihn „nichts Heiliges an sich “, sie sind lediglich „Instrumente“ (1996, S. 126). Als Instrumente können sie unter Umständen ihre Funktionalität verfehlen, mitunter gar kontraproduktiv werden. Ihre Brauchbarkeit muss sich immer wieder in der demokratischen Praxis beweisen. Verfehlen sie diesen Test jedoch dauerhaft, muss nach Alternativen geschaut werden:

„Die Bildung von Staaten muß ein experimenteller Prozeß sein. [...] Und weil die Bedingungen des Handelns und der Untersuchung und des Wissens immer wechseln, muß das Experiment immer wieder aufgenommen werden; der Staat muß immer wieder neu entdeckt werden“ (Dewey 1996, S. 42).

Wie gesehen, können gegenwärtig weder das allgemeine Wahlrecht noch unkonventionelle Beteiligungsformen die Normen der politischen Gleichheit und der sozialen Demokratie erfüllen. Diese postdemokratische Konstellation hat dazu motiviert, nach alternativen Institutionen zu suchen. Leitend für diese Suche ist die Einsicht von Young und McCormick gewesen, dass allgemein zugängliche Institutionen in Gesellschaften, die de facto durch ein nicht unerhebliches Maß an sozialer und auch kultureller Ungleichheit gekennzeichnet sind, dazu tendieren, die Interessen sozial starker Milieus zu privilegieren. Dies gilt, wenn auch in deutlich abgeschwächter Form, ebenso für Vorschläge, die sich durch eine Realisierung der deskriptiven Repräsentation mehr politische Gleichheit erhoffen. Dem soll durch die Einführung eines milieuspezifischen Vetorechtes begegnet werden. Allerdings handelt es sich bei diesem Vorschlag um eine Art Gedankenexperiment. Einer Umsetzung stehen nicht nur verfassungsrechtliche Prinzipien, sondern auch tiefverwurzelte Überzeugungen über den demokratischen Charakter des allgemeinen Wahlrechts entgegen. Fragen hinsichtlich des konkreten Designs dieses fakultativen Referendums kommen hinzu, etwa hinsichtlich der genauen Festlegung der Stimmberechtigten. Dennoch gibt es gute Gründe, der Intuition von Dewey zu folgen, dass politische Institutionen immer nur Instrumente zur Erreichung von Zielen sind, und angesichts der zu beobachtenden Schwächen der existierenden Mechanismen der politischen Entscheidungsfindung über alternative Verfahren nachzudenken. Dazu kann auch die Infragestellung grundlegender Institutionen und Verfassungsprinzipien wie dem der Allgemeinheit von Wahlen und Abstimmungen gehören, wenn sie denn die mit ihnen verbundenen Erwartungen nicht mehr erfüllen und unter Umständen ihnen sogar entgegenstehen. 


\section{Literatur}

Adams, John. 1776. Letter to John Penn. In The Works of John Adams, Vol. IV (18521865), 203-209. Boston: Little, Brown and Co.

Abromeit, Heidrun. 2002. Wozu braucht man Demokratie? Die postnationale Herausforderung der Demokratietheorie. Opladen: Leske \& Budrich.

Blühdorn, Ingolfur. 2013. Simulative Demokratie. Neue Politik nach der postdemokratischen Wende. Berlin: Suhrkamp.

Bobbio, Noberto. 1988. Die Zukunft der Demokratie. Berlin: Rotbuch.

Böhnke, Petra. 2011. Ungleiche Verteilung politischer und zivilgesellschaftlicher Partizipation. Aus Politik und Zeitgeschichte 61 (1-2): 18-25.

Borchert, Jens. 2003. Die Professionalisierung der Politik: zur Notwendigkeit eines Ärgernisses. Frankfurt/New York: Campus Verlag.

Buchstein, Hubertus. 2009. Demokratie und Lotterie. Das Los als politisches Entscheidungsinstrument von der Antike bis zur EU. Frankfurt/New York: Campus Verlag.

Buchstein, Hubertus, und Michael Hein. 2009. Zufall mit Absicht. Das Losverfahren als Instrument einer reformierten Europäischen Union. In Demokratie in der Weltgesellschaft, Soziale Welt Sonderband 18, Hrsg. Hauke Brunkhorst, 351-384. Nomos: Baden-Baden.

Buchstein, Hubertus, und Frank Nullmeier. (Hrsg.) 2006. Postdemokratie. Ein neuer Diskurs? Themenschwerpunkt. Forschungsjournal Neue Soziale Bewegungen 19 (4).

Christiano, Thomas. 1996. The Rule of the Many. Fundamental Issues in Democratic Theory. Boulder: Westview.

Crouch, Colin. 2004. Post-Democracy. Cambridge: Polity.

Dahl, Robert A. 1989. Democracy and its Critics. New Haven: Yale University Press.

Dahl, Robert A. 2006. Politische Gleichheit - ein Ideal? Hamburg: Hamburger Edition.

Dalton, Russell J. 2004. Democratic Challenges, Democratic Choices. The Erosion of Political Support in Advanced Industrial Democracies. Oxford: Oxford University Press.

Decker, Frank. 2011. Direkte Demokratie versus parlamentarisches Regierungssystem. Was sagen uns die Erfahrungen aus den Ländern? In Krise und Reform politischer Repräsentation, Hrsg. Markus Linden und Winfried Thaa, 217-237. Baden-Baden: Nomos.

Dewey, John. 1996. Die Öffentlichkeit und ihre Probleme. Bodenheim: philo.

Dörner, Andreas. 2002. Wahlkämpfe - eine rituelle Inszenierung des ,demokratischen Mythos'. In Wablkämpfe. Betrachtungen über ein demokratisches Ideal, Hrsg. Andreas Dörner und Ludgera Vogt, 16-42. Frankfurt a. M.: Suhrkamp.

Embacher, Serge. 2009. Demokratie! Nein danke? Demokratieverdruss in Deutschland. Die neue Studie der Friedrich-Ebert-Stiftung. Bonn: dietz.

Fishkin, James S. 1991. Democracy and Deliberation. New Directions for Democratic Reform. New Heaven: Yale University Press.

Geißel, Brigitte. 2011. Partizipative Innovationen auf lokaler Ebene - Chance oder Gefahr für die politische Repräsentation von Armen und Fremden. In Krise und Reform politischer Repräsentation, Hrsg. Markus Linden und Winfried Thaa, 195-216. BadenBaden: Nomos.

Geißel, Brigitte. 2012. Politische (Un-)Gleichheit. Aus Politik und Zeitgeschichte 62 (3839): 32-37.

Hay, Colin. 2007. Why We Hate Politics. Cambridge: Polity Press. 
Jörke, Dirk. 2010. Die Versprechen der Demokratie und die Grenzen der Deliberation. Zeitschrift für Politikwissenschaft 20: 269-290

Landemore, Hélène 2013. Democratic Reason. Politics, Collective Intelligence, and the Rule of the Many. Princeton: Princeton University Press.

Linden, Markus, und Winfried Thaa (Hrsg.). 2009. Die politische Repräsentation von Fremden und Armen. Baden-Baden: Nomos.

Linden, Markus und Winfried Thaa. 2011. Die Krise der Repräsentation - gibt es Auswege? In Krise und Reform politischer Repräsentation, Hrsg. Markus Linden und Winfried Thaa, 305-324. Baden-Baden: Nomos.

Keane, John. 2009. The Life and Death of Democracy. London: Simon \& Schuster.

Knaut, Annette. 2011. Die Quotenfrau: Erfolgsmodell (auch) für substantielle Repräsentation? In Krise und Reform politischer Repräsentation, Hrsg. Markus Linden und Winfried Thaa, 279-301. Baden-Baden: Nomos.

Manin, Bernard. 2007. Kritik der repräsentativen Demokratie. Berlin: Matthes \& Seitz.

McCormick, John P. 2011. Machiavellian Democracy. New York: Cambridge University Press.

Merkel, Wolfgang, und Alexander Petring. 2011. Partizipation und Inklusion. http://www. demokratie-deutschland-2011.de/partizipation-und-inklusion.php; zuletzt abgerufen am 27.03.2013.

Meyer, Thomas. 2009. Soziale Demokratie. Eine Einführung. Wiesbaden: VS Verlag für Sozialwissenschaften.

Michels, Robert. [1911] 1989. Soziologie des Parteiwesens in der modernen Demokratie. Untersuchungen über die oligarchischen Tendenzen des Gruppenlebens (4. Aufl.). Stuttgart: Kröner.

Mudde, Cas. 2007. Populist radical right parties in Europe. Cambridge: Cambridge University Press.

Nève, Dorothée de. 2009. NichtwählerInnen - eine Gefahr für die Demokratie? Opladen \& Farmington Hills: Verlag Barbara Budrich.

Nolte, Paul . 2012. Was ist Demokratie? Geschichte und Gegenwart. München: C. H. Beck.

Nordmann, Jürgen, Katrin Hirte und Walter O. Ötsch (Hrsg.) 2012. Demokratie! Welche Demokratie? Postdemokratie kritisch hinterfragt. Marburg: Metropolis.

Oesch, Daniel. 2008. Explaining Workers' Support for Right-Wing Populist Parties in Western Europe: Evidence from Austria, Belgium, France, Norway, and Switzerland. International Political Science Review 29: 349-373.

Pitkin, Hanna F. 1967. The Concept of Representation. Berkeley: University of California Press.

Phillips, Anne. 1993. Democracy and Difference. Cambridge: Polity Press.

Putnam, Robert D., und Susan J. Pharr (Hrsg.). 2000. Disaffected Democracies: What's Troubling the Trilateral Countries? Princeton: Princeton University Press.

Rosanvallon, Pierre. 2008. Counter-Democracy. Politics in an Age of Distrust. Cambridge: Cambridge University Press.

Rosanvallon, Pierre. 2010. Demokratische Legitimität. Unparteilichkeit - Reflexivität Nähe. Hamburg: Hamburger Edition.

Schaal, Gary S., und Claudia Ritzi. 2012. Deliberative Partizipation. Eine kritische Analyse des Verhältnisses von Deliberation, demokratischer Öffentlichkeit und staatlicher Ent- 
scheidung. In Partizipation und Staatlichkeit, Hrsg. Gisela Riescher und Beate Rosenzweig, 131-153. Suttgart: Steiner.

Schäfer, Armin. 2010. Die Folgen sozialer Ungleichheit für die Demokratie in Westeuropa. Zeitschrift für Vergleichende Politikwissenschaft 4: 131-156.

Schäfer, Armin. 2011. Republican Liberty and Compulsory Voting. MPIfG Discussion Paper 11/17.

Schäfer, Armin und Harald Schoen. 2013. Mehr Demokratie, aber nur für wenige? Der Zielkonflikt zwischen mehr Beteiligung und politischer Gleichheit. Leviathan 41: 94120.

Schmidt, Manfred G. 2008. Demokratietheorien. Eine Einführung (4. Aufl.).Wiesbaden: VS Verlag für Sozialwissenschaften.

Stone, Peter. 2010. The Luck of the Draw. The Role of Lotteries in Decision-Making. Oxford: Oxford University Press.

Streeck, Wolfgang. 2013. Gekaufte Zeit. Die vertagte Krise des demokratischen Kapitalismus. Berlin: Suhrkamp.

Thaa, Winfried 2009. Das ungelöste Inklusionsproblem in der partizipatorischen Neubewertung politischer Repräsentation. In Die politische Repräsentation von Fremden und Armen, Hrsg. Markus Linden und Winfried Thaa, 61-78. Baden-Baden: Nomos.

Thaa, Winfried 2013. Gruppenrepräsentation und demokratischer Prozess bei Iris Marion Young. In Zwischen Demokratie und globaler Verantwortung. Iris Marion Youngs Theorie politischer Normativität, Hrsg. Peter Niesen, 187-206. Baden-Baden: Nomos.

Vester, Michael. 2009. Soziale Milieus und die Schieflagen politischer Repräsentation. In Die politische Repräsentation von Fremden und Armen, Hrsg. Markus Linden und Winfried Thaa, 21-59. Baden-Baden: Nomos.

Walter, Franz. 2009. Im Herbst der Volksparteien? Eine kleine Geschichte von Aufstieg und Rückgang politischer Massenintegration. Bielefeld: transcript.

Winters, Jeffrey A. 2011. Oligarchy. New York: Cambridge University Press.

Young, Iris. M. 1990. Justice and the Politics of Difference. Princeton: Princeton University Press.

Young, Iris. M. 1997. Deferring Group Representation. In Ethnicity and Group Rights, Hrsg. Ian Shapiro und Will Kymlicka, 349-376. New York: New York University Press. Young, Iris. M. 2000. Inclusion and Democracy. Oxford: Oxford University Press.

\section{Autorenangaben:}

PD Dr. Dirk Jörke,

Universität Greifswald, Institut für Politik- und Kommunikationswissenschaft, Baderstr.

4-7, 17487 Greifswald, joerke@uni-greifswald.de 